\title{
Dielectric Relaxation in Methanol-Acetonitrile Mixtures
}

\author{
M. Stockhausen and E. Dachwitz \\ Institut für Physikalische Chemie der Universität Münster
}

Z. Naturforsch. 39a, 646-650 (1984); received April 2, 1984

\begin{abstract}
The complex permittivity of methanol + acetonitrile at $20^{\circ} \mathrm{C}$ was measured over the whole composition range at frequencies up to $36 \mathrm{GHz}$. It can be described by superposition of two Debye type components, one of them being due to associated methanol. This component is discussed with respect to two simple models, considering relaxation as governed by rotational tumbling of associates or by fluctuation of hydrogen bonds. The latter description appears to be the more suitable one.
\end{abstract}

\section{Introduction}

The complex permittivity of aliphatic alcohols is among the subjects investigated since the early stages of dielectric relaxation research [1]. The pure liquids generally exhibit a dominant absorption region, followed by a second one at higher frequencies $[2,3]$. Only the latter remains in diluted solutions, thus being evidently due to the relaxation of individual alcohol molecules. It can further be subdivided into spectral components which are to be ascribed to the reorientation of the $\mathrm{OH}$ moment components. These should be considered together with the far infrared absorption features [4]. The main absorption of neat alcohols, consequently, must be related to the well-known molecular association via hydrogen bonds in these liquids.

Studies of more concentrated solutions, which have been performed too, using both polar and nonpolar solvents [5-9], offer the opportunity to gather information on the association process, so far as it is observable by dielectric parameters at all. In the present paper, we wish to report results on such a system with a polar solvent, that is the binary mixture methanol-acetonitrile. It was chosen because of the similarity of the components with respect to molecular size and viscosity, while their relaxation times differ appreciably by about an order of magnitude. Perhaps this system is exemplary of further mixtures of polar components, whereof one is associative. At least methanol and other nitriles, as well as water and acetonitrile, which we have examined too, show quite the same

Reprint requests to Prof. M. Stockhausen, Institut für Physikalische Chemie der Universität, Schlossplatz 4, D-4400 Münster (FRG). dielectric behaviour. We shall focus our attention in particular on the associate relaxation contribution, in order to discuss whether it can be understood from the prevalently taken classical viewpoint, assuming rotational reorientation of definite entities [10], or whether an alternative description could be appropriate.

\section{Experimental and Results}

Complex permittivity measurements were carried out at 6 spot frequencies in the range from $30 \mathrm{MHz}$ to $36 \mathrm{GHz}$ with an accuracy of a few percent, using resonator methods for the most part. The conductivity contribution, if significant, was subtracted from $\varepsilon^{\prime \prime}$. The temperature was maintained at $20^{\circ} \mathrm{C}$.

Results were regarded particularly in terms of $\varepsilon^{\prime \prime}$. Presented here are the relaxation parameters $\tau_{i}, S_{i}$ as obtained by fitting a sum of elementary Debye type spectral components to the experimental data,

$$
\varepsilon^{\prime \prime}(\omega)=\Sigma \frac{S_{i} \omega \tau_{i}}{1+\omega^{2} \tau_{i}^{2}},
$$

where the least acceptable number of components was taken. In all cases, the data could be approximated within their uncertainty range by two components (one component for neat nitrile only). The formal procedure eventually results in relaxation times $\tau_{2}$ (where $\tau_{2}<\tau_{1}$ ) which are too short to define the respective spectral component as essentially within the experimental frequency range. In those cases, the variability range of parameters was used to choose longer $\tau_{2}$ times.

The total dispersion step derived from this analysis, $\Sigma S_{i}$, was checked against the difference of static permittivity and squared refractive index,

0340-4811/ 84 / 0700-0646\$01.30/0. - Please order a reprint rather than making your own copy. 


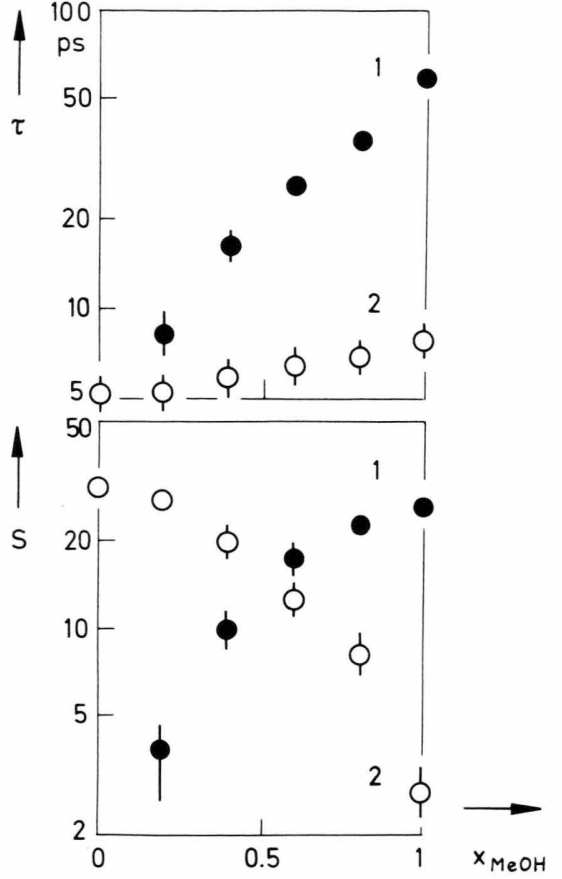

Fig. 1. Experimental relaxation parameters $\tau_{1,2}$ and $S_{1,2}$ as depending on the mole fraction of methanol, $x_{\mathrm{MeOH}}$.

$\varepsilon_{s}-n^{2}$. Generally, we had $\left(\varepsilon_{s}-n^{2}\right)-\Sigma S_{i}=2 \ldots 4$, which is the normal finding when higher frequency measurements are lacking and the respective relaxation contributions are disregarded.

The results obtained in that way are represented in Figure 1.

\section{Discussion}

The features of Fig. 1 suggest that the two formally obtained spectral components can in fact be attributed to certain distinguishable relaxing species.

Spectral component 1 is obviously related to methanol, as seen from its absence in neat nitrile and from the interchange of the dominant role between components 1 and 2. Moreover, this component is likely to be related to associated methanol, since it tends towards the corresponding low frequency component of neat methanol. It is this component we shall draw our attention to in course of the further discussion.

Component 2 is likely to be related to "free" molecules (monomers) of both kinds, alcohol as well as nitrile, since the relaxation time $\tau_{2}$ is in the order to be expected for molecules of the respective size, undergoing rotational tumbling at the given viscosity (which, besides a very weak minimum, changes monotonously over the mixture range [11]). In detail, two absorption terms should therefore contribute to component 2. However, these will be undistinguishable due to the limited range of high frequency measurements. An according analysis of experimental data is nevertheless possible, assuming two superimposed Debye type contributions to component 2 (thus disregarding further high frequency details). Especially this can be done with fixed values $\tau_{2}(\mathrm{AN})$ and $\tau_{2}(\mathrm{MeOH})$ by taking $S_{2}$ (AN) as proportional to the nitrile volume fraction. It is therefore very likely that the contribution of nitrile to the overall relaxation is concentrated in component 2. This substantiates the above supposition that component 1 is merely due to methanol. With respect to the alcohol association, nitrile behaves as quasi inert solvent, meaning here that heteroassociation need not be taken into account.

We turn now upon component 1 again with the aim to get some more insight into the relaxation behaviour of the methanol associates. Its relaxation time $\tau_{1}$ will be the main point of discussion. For that purpose, two model conceptions will be regarded which emphasize two extreme aspects of one and the same microdynamical process.

\section{Relaxation governed by tumbling of associates}

Put the case that methanol polymers are long lived and behave as quasi rigid entities, obeying the classical Debye relaxation mechanism via rotational tumbling. Then the relaxation time is determined by the complex size (at given temperature and viscosity), so that a distribution of relaxation times is to be expected whenever entities of different association number are present. To get a rough estimation of the effect of size on relaxation time, we refer to results obtained with rigid molecules in nonpolar solvents [12]. From these, one expects for the present system an approximately exponential increase of relaxation time by a factor of 2 on a difference of the effective radius of the tumbling entity of only 0.05 to $0.1 \mathrm{~nm}$. This is less than the radius increase on stepwise addition of a further molecule to any polymer. To understand the relaxation time of pure methanol, no more than about 6 molecules would be needed for a relaxing entity. 
Experimentally, two spectral components with a relaxation time ratio of 2 should be discernible by a clear deviation from Debye type absorption. Nothing of that kind is observed in the low frequency spectral component of the present system. Acceptance of the model would consequently require the entities to have a definite size and association number for their vast majority.

On the other hand, the increase of $\tau_{1}$ with increasing methanol content requires a gradual variation of the association number. Here we encounter the "classical" problem how to interprete the occurance of strict Debye type absorption in liquids with strong molecular interactions. To circumvent this difficulty one should reasonably look for a relaxation model that does not involve the size of the relaxing entity as essential parameter.

\section{Relaxation governed by fluctuations} of hydrogen bonds [13, 14]

Consider an individual alcohol molecule. It may tumble as a "free" molecule, until it is linked to another one or to a polymer by a hydrogen bond. For simplicity we assume that the molecule in this associated state will be motionless (which is not an indispensable condition). After the lifetime of the bond, the molecule will return into the "free"state, and so on.

The two state model is, in that form, oversimplified, but can nevertheless serve as guiding line to a modified interpretation of relaxation parameters.

To achieve a convenient treatment of the model, we shall consider the time correlation function of a certain component of a unit vector, which we assume to be fixed on the molecule parallel to its electric moment. Let $y(t)$ be that component and $\Psi(t)=\langle y(0) y(t)\rangle$ the correlation function. Since an exponential time correlation $\Psi(t)$ is equivalent to a Debye type single particle contribution to the permittivity, viz. $\varepsilon^{\prime \prime}(\omega)$, it is sufficient to regard $\Psi(t)$ with respect to whether or not it is of the exponential type.

The stochastic time dependence of $y(t)$ may be described in the following manner. The fluctuation in the "free" state, in case it would not be interrupted by associated states, is presupposed to exhibit an exponential correlation function with correlation time $\tau_{\mathrm{f}}^{*}$. The duration of the "free" phases is assumed to be exponentially distributed, with the characteristic time $T_{\mathrm{f}}$. In the associated state, $y(t)$ remains constant for a time which again is presupposed to have an exponential distribution, the characteristic lifetime being $T_{\mathrm{a}}$ in that case.

Weight factors (fractions) $\gamma_{i}$ of both states can be defined on the time scale by writing

$$
\gamma_{\mathrm{i}}=\frac{T_{\mathrm{i}}}{T_{\mathrm{f}}+T_{\mathrm{a}}} \quad(\mathrm{i}=\mathrm{f}, \mathrm{a}) .
$$

The model implies that for $\gamma_{\mathrm{f}} \rightarrow 1$ or $\gamma_{\mathrm{a}} \rightarrow 1$ the respective amplitude distributions become equal. The mean squared amplitude for these limiting cases will be denoted by $\left\langle y^{2}\right\rangle$.

Formally, the process $y(t)$ can be written as sum of two parts,

$$
y(t)=y_{\mathrm{f}}(t)+y_{\mathrm{a}}(t),
$$

where $y_{\mathrm{f}}(t)=y(t)$ in the "free" and $y_{\mathrm{f}}(t)=0$ in the associated phase. The complementary holds for $y_{\mathrm{a}}(t)$. Part " $\mathrm{f}$ ", considered as an isolated and autonomous process, has a correlation function

$$
\Psi_{\mathrm{f}}(t)=\gamma_{\mathrm{f}}\left\langle y^{2}\right\rangle e^{-t / \tau_{\mathrm{f}}}
$$

with

$$
\tau_{\mathrm{f}}^{-1}=\tau_{\mathrm{f}}^{*-1}+T_{\mathrm{f}}^{-1} .
$$

Part "a", on the assumptions given above, has

$$
\Psi_{\mathrm{a}}(t)=\gamma_{\mathrm{a}}\left\langle y^{2}\right\rangle e^{-t / T_{\mathrm{a}}} .
$$

Now the resulting correlation function $\Psi(t)$ of the complete process $y(t)$ is easily obtained for two limiting cases:

I) If $T_{\mathrm{f}}, T_{\mathrm{a}} \ll \tau_{\mathrm{f}}^{*}$, the resulting correlation function is a single, dilated exponential

$$
\Psi(t)=\left\langle y^{2}\right\rangle e^{-t / \tau}
$$

with

$$
\tau=\tau_{\mathrm{f}}^{*} / \gamma_{\mathrm{f}} .
$$

II) If $T_{\mathrm{f}}, T_{\mathrm{a}} \gg \tau_{\mathrm{f}}^{*}$, cross correlation between $y_{\mathrm{f}}(t)$ and $y_{\mathrm{a}}(t)$ becomes negligible, and the resulting correlation function is

$$
\Psi(t)=\Psi_{\mathrm{f}}(t)+\Psi_{\mathrm{a}}(t),
$$

thus, according to (4) and (6), the sum of two exponentials.

In the intermediate region, $\Psi(t)$ will generally be of non-exponential form. However, it may be approximated by a single exponential or by a sum of 
two exponentials as

$$
\Psi(t) \approx\left\langle y^{2}\right\rangle \sum_{1,2} g_{i} e^{-t / \tau_{i}} .
$$

Numerical calculations on model correlation functions show that the apparent parameters, viz. $\tau_{i}, g_{i}$ in (10), obey to some extent a simple extrapolation from the limiting cases.

Approximately, $\Psi(t)$ remains a single exponential as far as $T_{\mathrm{a}}<\tau_{\mathrm{f}}^{*} / \gamma_{\mathrm{f}}$, with the apparent $\tau$ according to (8). The weight factor is necessarily $g=1$ in that case.

On the other hand, for $T_{\mathrm{a}}>\tau_{\mathrm{f}}^{*} / \gamma_{\mathrm{f}}$ there are two exponentials in the approximation of (10). Their correlation times follow the limiting behaviour as if (9) would still hold, that means

$$
\tau_{1}=T_{\mathrm{a}} ; \quad \tau_{2}=\tau_{\mathrm{f}}^{*} .
$$

However, the apparent weight factors $g_{1,2}$, which in the limiting case equal $\gamma_{\mathrm{a}, \mathrm{f}}$, respectively, change monotonously when $T_{\mathrm{a}} \rightarrow \tau_{\mathrm{f}}^{*} / \gamma_{\mathrm{f}}$, so that

$$
g_{1} \rightarrow 1 ; \quad g_{2} \rightarrow 0
$$

These results are summarized in Figure 2.

We conclude that, within the framework of the model, the one particle correlation function will be of the one or two exponentials type, depending whether the lifetime of the associates is shorter or longer than the value $\tau_{\mathrm{f}}^{*} / \gamma_{\mathrm{f}}$. While in the first case the apparent correlation time is mixed from "free" and associated state characteristic times, the two apparent correlation times in the latter case have simple meanings as associate lifetime and "free" tumbling correlation time.

To proceed to the situation of dielectric relaxation measurements, the moment fluctuations of the macroscopic sample become the quantity to be taken into consideration. Molecular dipole moments may be introduced as simply proportional to the unit vector regarded above. The summation of molecular moments to the total moment, however, may involve problems, e.g. since at least two individual association states must be correlated to represent a hydrogen bond. Nevertheless the characteristics of the one particle correlation function will cum grano salis appear in the macroscopic correlation function, so that the above considerations may serve as a starting point for at least a qualitative discussion.

Accordingly, the experimental results, as far as methanol is concerned, are indicative of the two
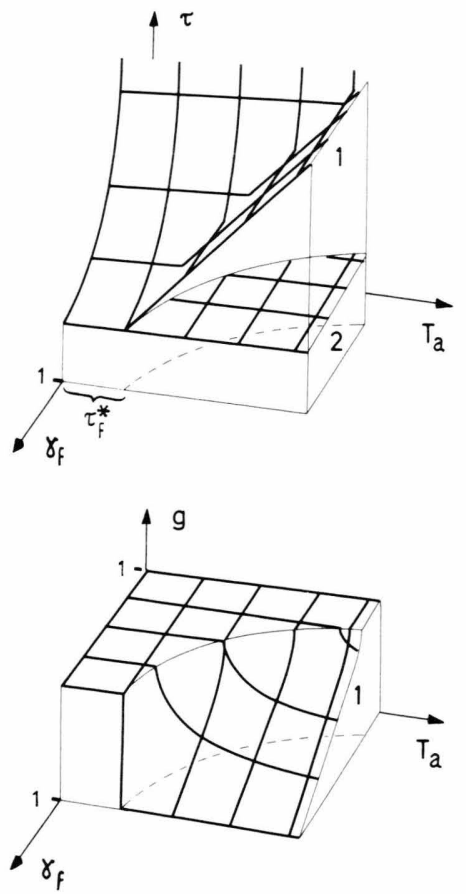

Fig. 2. Qualitative sketch of the approximate correlation function parameters $\tau_{1,2}$ and $g_{1}$ according to (10), as depending on the model parameters fraction of "free" methanol $\gamma_{\mathrm{f}}$ and lifetime of associate $T_{\mathrm{a}} \cdot g_{2}$ is not drawn since it is complementary to $g_{1}\left(g_{2}=1-g_{1}\right)$.

exponentials situation occuring for $T_{\mathrm{a}}>\tau_{\mathrm{f}}^{*} / \gamma_{\mathrm{f}}$. Thus $\tau_{1}$ of Fig. 1 should represent the lifetime of a molecule in the associated state, as depending on the mixture composition. It decreases with decreasing methanol content and tends towards $\tau_{2}$. (Figure 1 seems to indicate that $\tau_{1} \rightarrow \tau_{2}$ for $x_{\mathrm{MeOH}} \rightarrow 0$, but this cannot be stated with certainty since the resolution of spectral components is poor in that region.) The observed trend means that in Fig. 2 a point near $T_{\mathrm{a}} \approx \tau_{\mathrm{f}}^{*} ; \gamma_{\mathrm{f}} \approx 1$ will be reached. Consequently, the fraction $\gamma_{\mathrm{a}}$ of associated methanol should become small on dilution, which is a reasonable result.

Some additional information may be obtained from the parameter $S_{1}$. If $v_{\mathrm{MeOH}}$ is the volume fraction of methanol in the mixture, $\mu_{\mathrm{a}}$ the mean total moment of an associate, $n_{\mathrm{a}}$ the mean association number, then the proportionality

$$
S_{1} / v_{\mathrm{MeOH}} \sim \gamma_{\mathrm{a}} \mu_{\mathrm{a}}^{2} / n_{\mathrm{a}}
$$

should hold. Let us regard the behaviour on dilution as seen by extrapolation from the range of 
higher methanol contents $\left(x_{\mathrm{MeOH}}>0.2\right)$. The experimental $S_{1} / v_{\mathrm{MeOH}}$ values do not vary significantly over that range. We have concluded before that $\gamma_{\mathrm{a}}$ becomes small, and since $\mu_{\mathrm{a}}$ will be finite, it follows from (13) that the association number $n_{\mathrm{a}}$ must become small. Thus we arrive again at the conclusion that the polymer size decreases on dilution, but this inference is not directly to be drawn from the $\tau_{1}$ behaviour, as it had been with the former model.

[1] S. Mizushima, Physik, Z. 28, 418 (1927).

[2] S. K. Garg and C. P. Smyth, J. Phys. Chem. 69, 1294 (1965).

[3] J. M. Fornies Marquina, F. J. Arcega, Y. Dutuit, G. Vicq, and A. M. Bottreau, Anal. Fis. B 77, 57 (1981).

[4] J. K. Vij, C. J. Reid, and M. W. Evans, Mol. Phys. 50, 935 (1983).

[5] D. J. Denney, J. Chem. Phys. 30, 1019 (1959).

[6] G. Klages and K. Obermayer, Z. Naturforsch. 18 a, 1125 (1963); G. Klages and N. Roth, Z. Naturforsch. 23 a, 171 (1968).

[7] S. K. Garg and P. K. Kadaba, J. Phys. Chem. 69, 674 (1965).

[8] T. Koshii, H. Takahashi, and K. Higasi, Bull. Chem. Soc. Japan 48, 993 (1975).

\section{Conclusions}

While in the first model the Debye character of the associate absorption is based on a definite polymer size, it rests on an exponential lifetime distribution in the second model. The latter seems to allow for a more natural interpretation of the alcohol associate relaxation, particularly in view of the usually accepted picture of hydrogen bonding networks.

[9] D. Bertolini, M. Cassettari, and G. Salvetti, J. Chem. Phys. 78, 365 (1983).

[10] D. Kivelson and P. A. Madden, J. Phys. Chem. 87, 4823 (1983).

[11] Landolt-Börnstein, Zahlenwerte und Funktionen, Vol. II/5 a. Springer-Verlag, Berlin 1969.

[12] F. Hufnagel, Z. Naturforsch. 25 a, 1143 (1970); F. Hufnagel and Shi Ding He, Z. Naturforsch. 36 a, 505 (1981).

[13] E. Bauer and M. Magat, Bull. Soc. Chim. Fr. 1949, 341.

[14] C. Brot, M. Magat, and L. Reinisch, Koll.-Z. 134, 101 (1953); C. Brot, Ann. Phys. Paris (13)2, 714 (1957). 